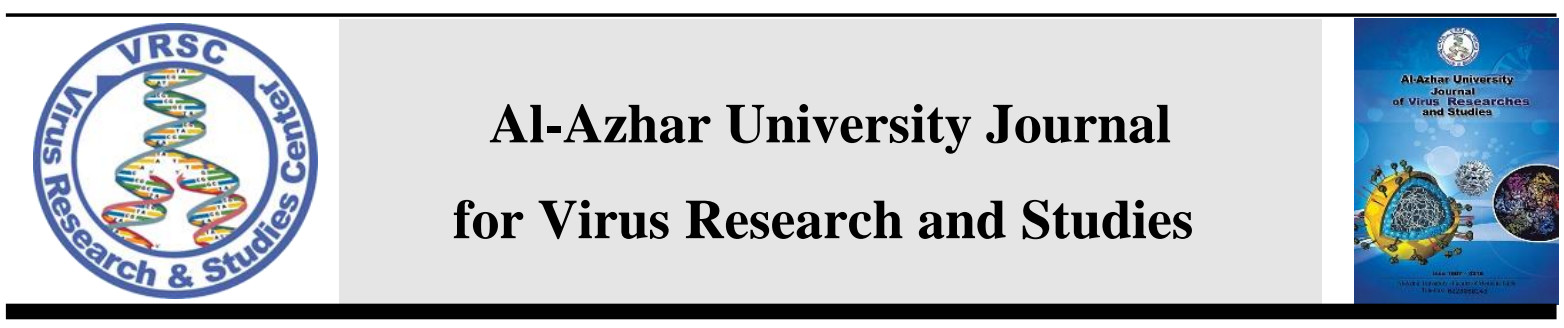

\title{
Impact of Calcium Channel Blockers on Murine Trichinellosis
}

Eman Mohammed Mahmoud*1, Saedia Abdel Hady Sayed El-Ahl ${ }^{1}$, Amal Abdelaziz El shafae $^{1}$, Amany Ahmed Abd Elaal ${ }^{2,3}$, Mohammed Sharaf El din Zaky Badr ${ }^{4}$, Khadyga Hussein Abdel Fadil ${ }^{1}$, Yasmine Fathy Elesawy ${ }^{5}$ and Magda Said Ahmed Abdeltawab ${ }^{2}$

${ }^{1}$ Department of Medical Parasitology, Faculty of Medicine for Girls, Al-Azhar University, Cairo, Egypt

${ }^{2}$ Department of Medical Parasitology, Faculty of Medicine, Cairo University, Cairo, Egypt

${ }^{3}$ Department, of Medical Parasitology, Faculty of Medicine, Armed Forces College of Medicine (AFCM)

${ }^{4}$ Department of Molecular Biology, Medical Research Centre, Faculty of Medicine, Ain Shams University Cairo, Egypt

${ }^{5}$ Department of Pathology, Faculty of Medicine, Cairo University, Cairo, Egypt

*E-mail: eman_omar162011@yahoo.com

\begin{abstract}
Essentially, Trichinella spiralis is considered to be a re-emerging intestinal and tissue parasitic nematode that causes serious foodborne infections worldwide. To evaluate the role of a commonly used Calcium Channel Blocker (CCB) (nifedipine) on muscular phase of murine trichinellosis utilizing parasitological and histopathological parameters. The study was carried out on 45 Swiss albino mice. The infection was done orally for the infected groups with about $250 \pm 50$ Trichinella spiralis larvae (TSL). Sacrification of mice was done on the 35th day postinfection (PI). Detection and counting the number of larvae in the diaphragm of mice of each group were done microscopically and specimens were taken and fixed in $10 \%$ formalin to detect histopathological changes. The study revealed that nifedipine has reduction rate (67.96\%) on Trichinella larvae count in diaphragm. Histopathological examination revealed mild reduction of inflammatory reactions. Nifedipine has been concluded to be used in the treatment of trichinellosis, to reduce the number of larvae and to improve pathological changes.
\end{abstract}

Keywords: Trichinella spiralis, CCB (nifedipine), Muscular phase.

\section{Introduction}

Trichinellosis, a parasitic disease caused by Trichinella spp., most often Trichinella spiralis ( $T$. spiralis), is a globally distributed, re-emerging zoonosis [1]. In 55 countries worldwide, human trichinellosis has been reported and is a major socio-economic burden in some developing countries [2, 3]. In Egypt, human trichinellosis is present; the prevalence of $T$. spiralis larvae infection in 
slaughtered pigs was $1.08 \%$ in Albasatin slaughterhouse, Cairo Governorate (Egypt) [4]. Severity of the clinical picture depends on the species of the parasite, the number of living larvae ingested, the host's age, sex and immune status. There are intestinal, parenteral and muscular phases of trichinellosis, characterized by fever, malaise, anorexia, nausea, vomiting, abdominal pain and diarrhea, which may be so severe that increased mortality due to dehydration [5]. The striated muscles are mainly the muscles affected during the parenteral phase. Dyspnea, respiratory failure, myocarditis, cardiac failure and diffuse encephalopathy can be complicated [6]. In being the only species that occupies skeletal muscle fibers and modifies them into nurse cells, $T$. spiralis has a unique nature among all known nematodes. The parasite needs to recruit new blood vessels to the developing nurse cell in order to maintain a long-term host-parasite relationship and maintain its metabolic activity to meet the ever-growing demand for nutrients [7]. This is accomplished by initiation of angiogenesis this [8]. In all vascularized organs, pericytes are perivascular cells found in abundance. They control numerous functions, including the growth of vessels and angiogenesis [9]. Raza et al. (2010) [10] reported that angiogenesis modulation through the promotion of endothelial cell survival and migration is the main function of the pericyte in both skeletal and cardiac muscle. Expression of CD34 and CD31 has been used to identify and isolate pericytes from skeletal muscle [11, 12]. These markers have also been shown, more specifically, to induce therapeutic angiogenesis in animal models. In addition, $T$. spiralis infection induces the production of Vascular Endothelial Growth Factor (VEGF), which is a potent angiogenic stimulator. Both VEGF mRNA and VEGF peptides were detected in the developing nurse cell cytoplasm [13]. Moccia et al. [14] showed that the flux of calcium ions has many biological functions that promote angiogenesis and modulate it. Its effect on angiogenesis may change the environment needed for the formation of Trichinella nurse cells. Furthermore, in the synthesis and release of inflammatory chemical mediators, calcium ions play a significant role $[15,16]$. Several antiparasitic drugs are used to eliminate adults in the intestinal stage. Benzimidazoles, the most famous of which is mebendazole, are the most common anthelmintics. For children less than 2 years old, benzimidazole derivatives are not recommended and are poorly absorbed from the gastrointestinal tract [17]. Moreover, bioavailability, high degree of resistance and weak activity against encapsulated larvae are limiting factors [18]. Nifedipine is a blocker of the calcium channel that induces vaso-relaxation by blocking the influx of calcium ions into cells of vascular smooth muscle. It is used to decrease systemic blood pressure and to prevent coronary vasospasm by enhancing the functions and number of circulating endothelial progenitor cells that affect new vessel formation; nifedipine improves vascular endothelial function in hypertensive patients [19, 20]. In the present study, the impact of nifedipine as one of CCB on muscular phase (muscle of diaphragm) of murine trichinellosis was assessed by parasitological and histopathological parameters.

\section{Materials and Methods}

\subsection{Experimental animals:}

The present study was conducted on 45 males, laboratory bred Swiss albino mice, pathogen-free, 20-25 g weight and 4-6 weeks age, obtained from Theodor Bilharz Research Institute (TBRI), Cairo. The mice were kept on a standard diet under a temperature of $24 \mathrm{C}^{\circ}$. The study was approved by the ethical committee of the Faculty of Medicine for Girls, Al-Azhar University and by the ethical community of animal research at TBRI and was 
conducted in accordance with the international guidelines.

\subsection{Experimental design:}

The mice were divided into three main groups. Group (I): non-infected control group containing 15 mice. Group (II): infected non-treated group containing 15 mice. Group (III): infected group treated with nifedipine containing 15 mice.

\subsection{Experimental infection:}

Each mouse was infected orally with about $250 \pm 50 \quad T$. spiralis infective larvae, Egyptian strian (TSL) obtained from (TBRI) $[21,22,23]$.

\subsection{Drug administration:}

Nifedipine (Epilat, 20mg tablets, EPICO, Egypt) was dissolved in distilled water and the doses were calculated by extrapolation of human therapeutic doses to animal doses [24]. Nifedipine was administered on day one (PI) till day 35 (PI) at a dose of $80 \mu \mathrm{g}$ /mouse /day orally [25].

\subsection{Animal sacrification:}

Sacrification of mice was done, according to Denham, (1965) and Gamble, (1996) $[21,22]$. on day $35(\mathrm{PI})$ after completing the treatment, to determine the impact of the used drug on larvae in the muscular phase.

\subsection{The following parameters were evaluated}

\subsubsection{Parasitological examination:}

Detection and counting of muscle larvae: The diaphragm of infected non- treated and infected treated mice was carefully dissected in each mouse, examined under the microscope and the number of encysted larvae was counted per gram of muscle tissue according to Denham, (1965) and Gamble, [21, 22].

\subsubsection{Histopathological examination:}

Skeletal muscle specimens from the diaphragm were taken from mice sacrificed on $35^{\text {th }}$ day (PI) $[26,27]$. These specimens were fixed in $10 \%$ formalin, dehydrated and then embedded in paraffin blocks. Paraffin sections were cut into $5 \mu \mathrm{m}$ thickness, stained by haematoxylin and eosin [28] and examined microscopically to detect histopathological changes.

\subsection{Statistical analysis of collected data:}

Statistical analysis was done using SPSS program (statistical package of social science; SPSS Inc., Chicago, IL, USA) version 16 for Microsoft Windows. Median and range were calculated to measure central tendency and dispersion of quantitative data. Comparing groups was done using Kruskal-Wallis test to determine the significance in the difference between two medians.

\section{Results}

\subsection{Parasitological results:}

Thirty five days after treatment with nifedipine, number of T.spiralis larvae/g of muscle was 370 larvae/g indicating a 67.96 $\%$ reduction in comparison to the larval count in the infected non-treated group (GI), which was 1150 larvae/g. The reduction was of statistical significance at $\mathrm{P}<0.01$ (Table 1).

\subsection{Histopathological results:}

Histopathological examination of sections of diaphragm of the non- infected control group showed normal spindle shape muscle fiber with blue nuclei and pink matrix (Figure 1) while sections of diaphragm of the infected non-treated group showed encysted larvae surrounded with cellular infiltrate (Figure 2). In nifedipine-treated group, histopathological examination showed a mild reduction of 
inflammatory cellular infiltrate with intact larvae and capsule (Figure 3).

Table (1): Median and range of the number of T.spiralis larvae/g of muscle of mice in infected non-treated group versus nifedipine-treated group.

\begin{tabular}{||l|c|c|c|c||}
\hline \multirow{2}{*}{ Varieties } & $\begin{array}{c}\text { Number of } \boldsymbol{T} \text {. spiralis larvae/g of } \\
\text { muscle of different groups }\end{array}$ & \multirow{2}{*}{$\begin{array}{c}\text { Percentage } \\
\text { of } \\
\text { reduction }\end{array}$} & P value \\
\cline { 2 - 3 } Animal groups & \multicolumn{2}{|c||}{ Kruskal-Wallis test } & Range & \\
\cline { 2 - 3 } & Median & $800-1300$ & \multirow{2}{*}{$67.96 \%$} & $\mathbf{0 . 0 0 4} *$ \\
\hline \hline $\begin{array}{l}\text { Group II (infected non- } \\
\text { treated group) }\end{array}$ & 1150 & $\mathbf{2 7 0}-\mathbf{4 2 0}$ & & \\
\hline $\begin{array}{l}\text { Group III (infected treated } \\
\text { with nifedipine) }\end{array}$ & $\mathbf{3 7 0}$ & & \\
\hline
\end{tabular}

* Highly significant difference $(\mathrm{P}$ value $<0.01)$.

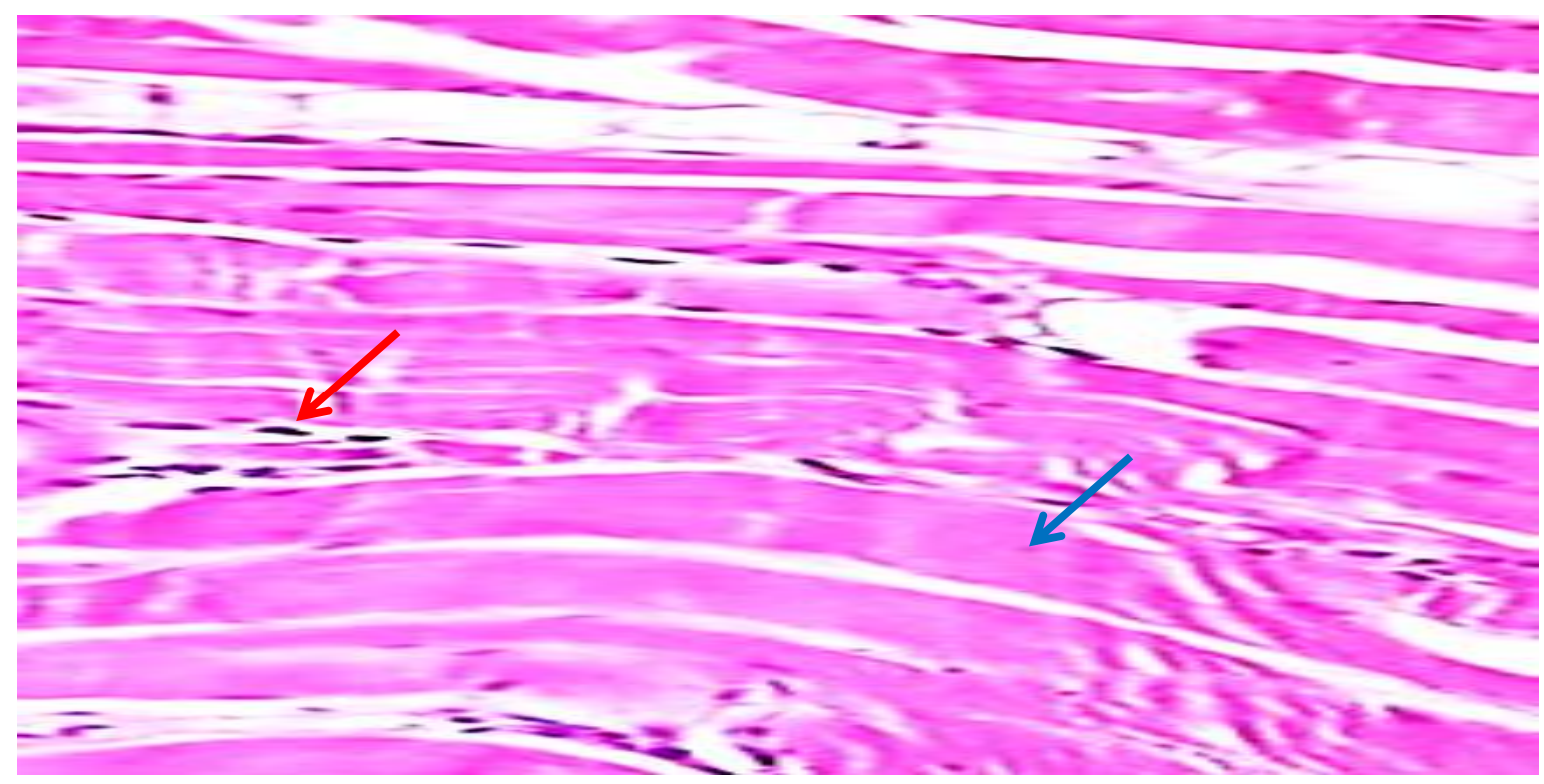

Figure (1): Non-infected control group (GI) showed normal spindle shape muscle fiber with blue nuclei (red arrow) and pink matrix (blue arrow) (H\&E 200x). 


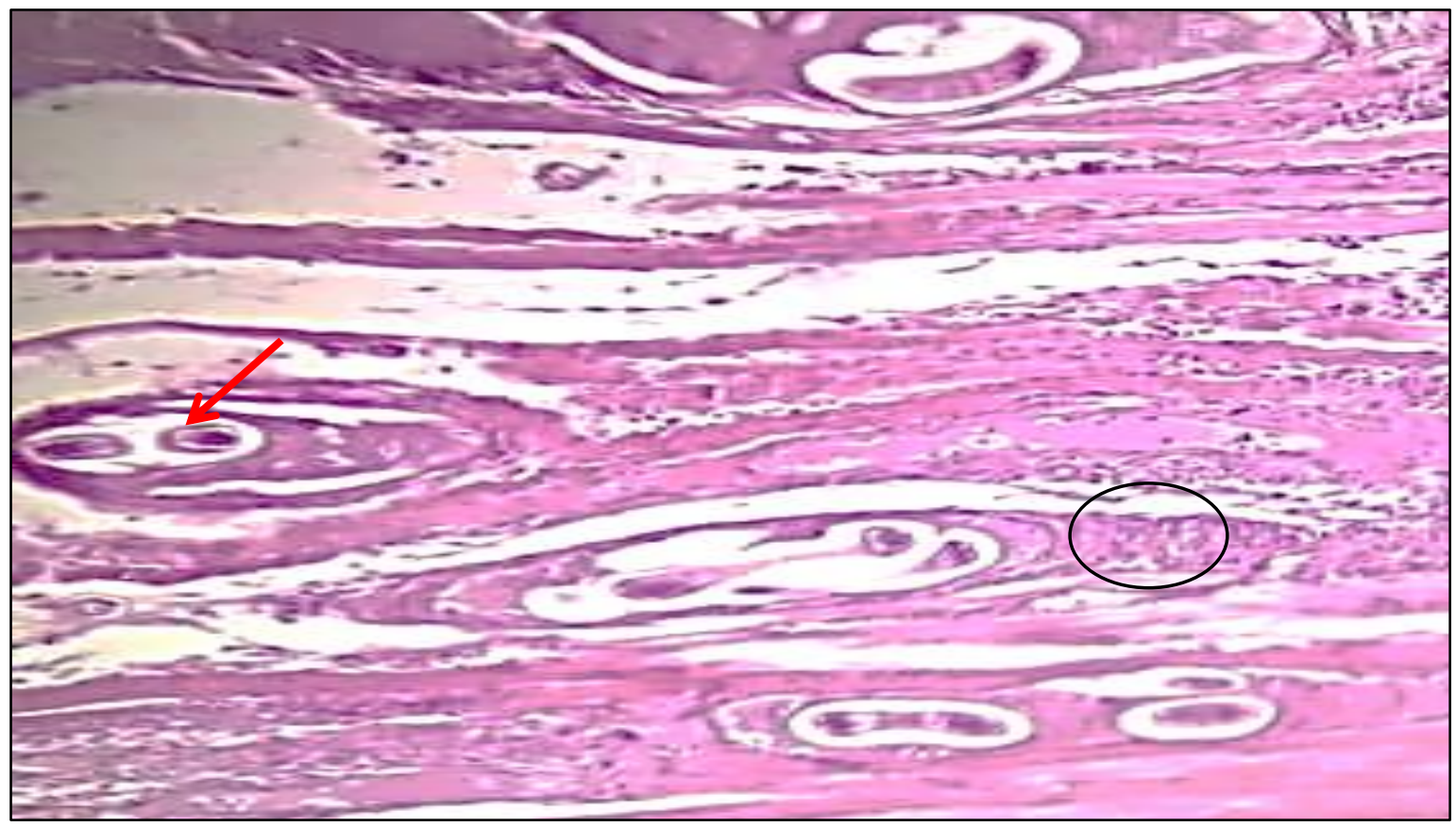

Figure (2): Infected non-treated group (GII) showing inflammatory cellular infiltration surrounding the encysted larvae including lymphocytes, eosinophils, macrophages and some neutrophils (black circle). Larvae were coated by a thick complete collagenous capsule with intact larval morphology (red arrow) (H\&E 200x).

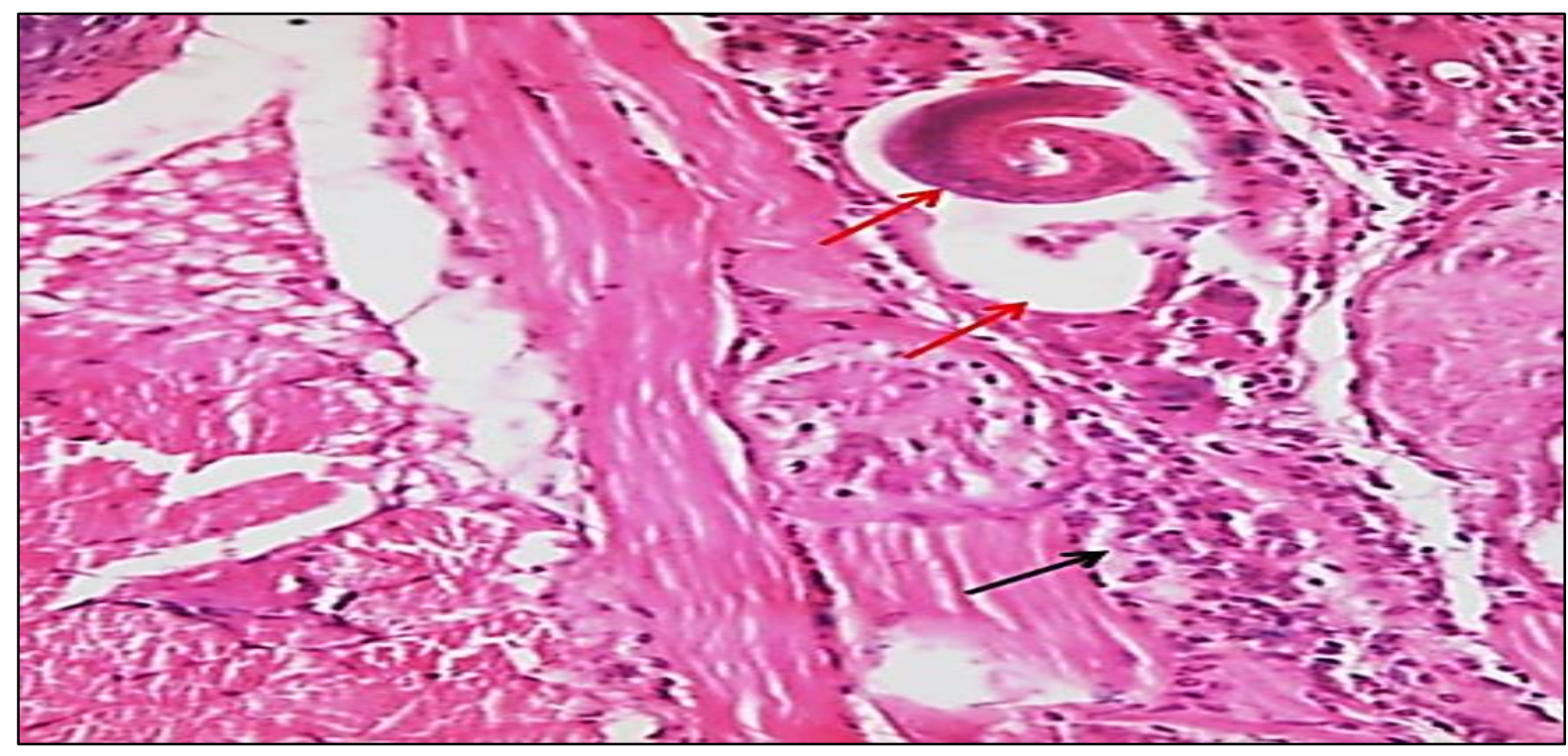

Figure (3): Infected nifedipine-treated group (GIII) showing a mild reduction in the intensity of inflammatory cellular infiltrate (black arrow) with apparently intact larvae and capsule (red arrow) (H\&E 200x). 


\section{Discussion}

Trichinellosis is a widespread, serious parasitic disease with significant implications for the medical, veterinary and economic sectors, particularly in developing countries [29].

In addition to occupying and developing inside the muscle cells, $T$. spiralis larvae transform it to be fully at their service after becoming nurse cells surrounded by a vascular network. Subsequently, not only does this network nourishes the parasite, but also protects it against the host immune response [8]. It has been reported that $T$. spiralis induces angiogenesis at the site of tissue infection to supply the required nutrients to the parasite and to act as a reliable waste disposal system by improving the expression during the formation of nurse cells of the chief angiogenic molecule, namely VEGF [8].

Ivermectin (IVM) is an alternative to benzimidazole and has anthelmintic options for the treatment of trichinellosis. It is a powerful macrocyclic lactone that, through increasing the influx of chloride ions across the cell membrane, causes paralysis in nematodes and arthropods [30]. In patients with encysted Trichinella larvae, the biochemical environment is affected by other drugs that may be used for the management of co-morbid conditions. Calcium channel blockers (CCB) that are widely used in clinical practice are among these medicines [31]. This research, to the best of our knowledge, is the first to study the effect of a CCB, nifedipine, on the muscular phase of experimental trichinellosis.

In skeletal muscles, calcium channels have an essential role and are important for their contraction. Calcium channel blockers have a direct relaxant effect on the skeletal muscle and decrease the strength of striated muscle contraction by inhibiting the release of calcium from the sarcoplasmic reticulum. In addition, calcium plays an important role in the contraction of blood vessels and angiogenesis [32]. Consequently, CCB may affect the necessary environmental conditions for the encystation of Trichinella larvae. Nifedipine was chosen in the current study to explore the effect of calcium channel blockade on the muscular phase of $T$. spirals infection. The effects of the drug on infection were evaluated and parasitological and histopathological effects were compared in various studied groups.

As regard to the parasitological assessment, nifedipine at a dose of $80 \mu \mathrm{g} / \mathrm{mouse} /$ day resulted in a substantial decrease in the number of $T$. spirals larvae in the diaphragm muscle compared to the control group, which was highly significant reduction $(67.96 \%)$.

These findings were in accordance with an in vitro study done by Christ and Stillson [33] who revealed that nematode muscle contraction appeared to depend on extracellular calcium of muscle membrane, and that nifedipine reduced the calcium influx across the muscle membrane of Acanthocheilonema viteae leading to inhibition of spontaneous isotonic contractions of the filariid parasite leading to flaccid paralysis.

Similar results were also observed in the study conducted by Silva-Moraes et al. [34] when nifedipine was added to adult Schistosoma mansoni cultures, an impaired or absent motility was observed in male and female worms together with several tegumental injuries. The authors attributed these observations to the blockage by nifedipine of calcium channels located on the external membrane of the Schistosoma mansoni, leading to flaccid paralysis of the parasite. Moreover, these findings can be attributed to the nifedipine-induced liberation of nitric oxide by host endothelial cells which plays a crucial role in anti-parasitic immunity and parasite damage [35].

In the current study, encysted larvae surrounded by inflammatory cellular infiltration including lymphocytes, eosinophils, macrophages and some neutrophils were revealed in the 
histopathological study. A thick full collagenous capsule with intact larval morphology in the infected group was revealed. These findings were similar to the findings recorded by Attia et al. (2015) [ 29] that showed the presence of a massive number of $T$. spiralis larvae diffusely present in muscles with massive number of chronic inflammatory cells surrounding the larvae.

For nifedipine-treated mice in the present study, histopathological examination of infected and treated mice showed mild improvement in the inflammatory reaction in muscle sections. The anti-inflammatory effects of CCBs were recorded by Chen $e t$ al. (1999) [36] and Kumar et al. (2016) [37] in an experimentally induced pleurisy and granuloma model in rats, where nifedipine was found to have anti-inflammatory effects.

The anti-inflammatory effects of CCBs have been attributed by Khaksari et al. [38]

\section{References}

1- Bai, X.; Hu, X.; Liu, X.; Tang, B. and Liu, M. (2017): Current research of trichinellosis in China. Front. J. Microbiol. 8: 1472-147.

2- Pozio, E. (2007): World distribution of Trichinella spp. infections in animals and humans. Vet. Parasitol. 149: 3-21.

3- Wang, M.; Wu, L.; Weng, R.; Zheng, $\mathrm{W}$; $\mathrm{Wu}, \mathrm{Z}$. and Lv, Z. (2017): Therapeutic potential of helminths in autoimmune diseases: helminth-derived immune-regulators and immune balance. Parasitol. Res. 116 (8):2065-2074.

4- Dyab, A.; Ahmed, M. and Abdelazeem, A.G. (2019): Prevalence and histopathology of Trichinella spiralis larvae of slaughtered pigs in to the inhibition of $\mathrm{Ca} 2+$ influx that plays a major role in the synthesis and release of inflammatory mediators. The reduction of $\mathrm{Ca} 2+$ inhibits the activity of phospholipase $\mathrm{A} 2$ and phospholipase $\mathrm{C}$, the enzymes responsible for leukotriene synthesis, and stabilizes the integrity of the cell membrane, thereby preventing injury and inflammation of the tissue.

\section{Conclusion}

Nifedipine; that widely used in cardiovascular diseases, can be used in the treatment of trichinellosis and has shown a high rate of reduction in muscle Trichinella larvae and improves pathology so that it can be used as an adjuvant therapy to treat trichinellosis. In order to achieve alternative therapeutic options for the management of this parasitic infection, further studies on their various types should be carried out.

Cairo Governorate, Egypt. J. Egypt. Soc. Parasitol. (JESP), 2019 (2), 439442.

5- Bruschi, F. and Murrell, K.M. (2002): New aspects of human trichinellosis: the impact of new Trichinella species Postgrad. Med. J. 78:15-22.

6- Yu, Y.U. and Yf, Q.I. (2015): Progress in Treatment and Prevention of Trichinellosis. J. Infect. Dis. Ther. 6 (3).

7- Patra, C. and Sarkar, S. (2014): Nurse cell Biology of Trichinella spiralis. I.J.O.A.R.T.4 (3): 2278-7763.

8- Ock, M.S.; Cha, H. and Choi, Y.H. (2013): Verifiable hypotheses for Thymosin $\beta 4$-dependent and independent angiogenic induction of Trichinella spiralis triggered nurse cell 
formation. Int. J. Mol. Sci. 14: 2349223498.

9- Cappellari, O. and Cossu, G. (2013): Pericytes in development and pathology of skeletal muscle. Circ. Res. J. 113:341-347.

10- Raza, A.; Franklin, M.J. and Dudek, A.Z. (2010): Pericytes and vessel maturation during tumor angiogenesis and metastasis. Am. J. Hematol. 85:593-598.

11- Campagnolo, P.; Cesselli, D.; Al Haj Zen, A.; Beltrami, A.P. and Krankel, N. (2010): Human adult vena saphena contains perivascular progenitor cells endowed with clonogenic and proangiogenic potential. circulation. 121:1735-1745.

12- Avolio, E.; Meloni, M.; Spencer, H.L.; Riu, F. and Katare, R. (2015): Combined intramyocardial delivery of human pericytes and cardiac stem cells additively improves the healing of mouse infarcted hearts through stimulation of vascular and muscular repair. Circ. Res. J. 116:81-94.

13- Alexander, R.; Mackie, Ph.D. and Douglas, W. (2011): CD34-Positive Stem Cells in the Treatment of Heart and Vascular Disease in Human Beings. Tex Heart Inst J.; 38(5): 474485.

14- Moccia, F.; Negri, S.; Shekha, M.; Faris, P.; 1, 2 and Guerra, G. (2019): Endothelial $\mathrm{Ca} 2+\quad$ Signaling, Angiogenesis and Vasculogenesis: Just What It Takes to Make a Blood Vessel. Intern. J. of Mol. Sci. 20(16), 3962.
15- Lucas, R.; Alves, M.; Del Olmo, E.; San, F. and Paya, M. (2003): A new in vitro inhibitor of intracellular calcium mobilization modulates acute and chronic inflammation. Biochem. Pharmacol. 65 (9):1539-1549.

16- Eteraf-Oskouei, T.; Mirak, S.M. and Najafi, M. (2017): Anti-Inflammatory and anti-Angiogenesis effects of verapamil on rat air pouch inflammation model. Adv. Pharm. Bull. 7 (4): 585-591.

17- Cañete, R.; Escobedo, A. A.; Almirall, P. et al. (2009): Mebendazole in parasitic infections other than those caused by soil-transmitted helminthes. Trop. Med. Hyg. 103: 437-442.

18- Caner, A.; Döşkaya, M.; Değirmenci, A.; Can, H. and Baykan, S. Y. (2008): Comparison of the effects of Artemisia vulgaris and Artemisia absinthium growing in western Anatolia against trichinellosis (Trichinella spiralis) in rats. Exp. Parasitol. 119: 173-179.

19- Lee, M.; Aoki, M.; Kondo, T.; Kobayashi, K.; Okumura. et al. (2005): Therapeutic angiogenesis with intramuscular injection of low-dose recombinant granulocyte-colony stimulating factor. Arterioscler Thromb. Vasc. Biol. 25:2535-2541.

20- Sugiura, T.; Kondo, T.; KureishiBando, Y.; Numaguchi, K.; Yoshida, O.et al. (2008): Nifedipine improves endothelial function role of endothelial progenitor cells. Amer. Heart. Asso. 11: 491-498. 
21- Denham, D.A. (1965): Studies with methyridine and Trichinella spiralis. I. Effect upon the intestinal phase in mice. Exp Parasitol; 17: 10-14.

22- Gamble, H.R. (1996): Detection of trichinellosis in pigs by artificial digestion and enzyme immunoassay. $\mathbf{J}$ Food Prot 59: 295-298.

23- Guenther, S.; Nöckler, K.; von Nickisch, M.; Landgraf, M.; Ewers, C.; Wieler, L.H. et al. (2008): Detection of $T$. spiralis $T$. britovi and $T$. pseudospiralis in muscle tissue with real-time PCR. J. Microboil. Methods. 75: 287-292.

24- Paget, G. E. and Barnes, J. M. (1964): Evaluation of results: Qualitative application in different species. In: Evaluation of drug activities. Pharmacometrics, Eds. Laurence, D. R. and Backarach, A. L. Academic press, London and New York.; 1: 160-167.

25- Lee, K.M.; Akang, H.; Ko, B.; Eun-Ha, O.H.; Park, M. et al. (2013): Differential gene expression profiles in spontaneously hypertensive rats induced by administration of enalapril and nifedipine. Intern. J. Mol. Med. 31: 179-187.

26- Zeromski, J.; Boczoń, K.; Wandurska, N. E. and Mozer, L. (2005): Effect of aminoguanidine and albendazole on inducible nitric oxide synthase (iNOS) activity in $\mathrm{T}$. spiralis-infected mice muscles. Folia. Histochem. Cytobiol. 43: 157-159.

27- Monib, M.E.M.; Shaheen, M.S.; Galal, L.A. and Farrag, H.M. (2010): Role of $T$. spiralis adult and larval antigens in immunomodulation of nitric oxide (NO) in intestinal and muscular phase of trichinelosis. Assiut Med J 34: 147158.

28- Shalaby, M.A.; Moghazy, F.M.; Shalaby, H.A. and Nasr, S.M. (2010): Effect of methanolic extract of Balanites aegyptiaca fruits on enteral and parenteral stages of Trichinella spiralis in rats. Parasitol. Res. J. 107: 17-25.

29- Attia, R.A.; Mahmoud, A.E.; Farrag, H.M.M. et al. (2015): Effect of myrrh and thyme on Trichinella spiralis enteral and parenteral phases with inducible nitric oxide expression in mice. Mem Inst Oswaldo Cruz, Rio de Janeiro, 110(8): 1035-1041.

30- Basyoni, M. M. and El-Sabaa, A. A. (2013). Therapeutic potential of myrrh and ivermectin against experimental Trichinella spiralis infection in mice. The Korean journal of parasitology, 51(3), 297-304.

31- Lee, C.W.; Sarna, S.K.; Singaram, C.et al. (2018): $\mathrm{Ca} 2+$ channel blockade by verapamil inhibits GMCs and diarrhea during small intestinal inflammation. American Physiological Society; 273: 785-794.

32- Zamponi, G.W.; Striessnig, J.; Koschak, A. and Dolphin, A.C. (2015): The Physiology, pathology, and pharmacology of voltage-gated calcium channels and their future therapeutic potential. Pharmacol Rev.; 67 (4):82170. 
33- Christ, D. and Stillson, T. (1992): Effects of calcium channel blockers on the contractility of the filariid Acanthocheilonema viteae. Parasitol Res.; 78(6):489-94.

34- Silva-Moraes, V.; Bubula Couto, F.F.; Vasconcelos, M.M.; Neusa, A. and Coelho, P.M. (2013): Antischistosomal activity of a calcium channel antagonist on schistosomula and adult Schistosoma mansoni worms. Mem. Inst. Oswaldo. J. 108(5): 600-604.

35- Bruschi, F. and Chiumiento, L. (2011): Trichinella inflammatory myopathy: host or parasite strategy? Parasites \& Vectors 42(4):1-6.

36- Chen, W.R.; Yang, Y.X.; Zheng, H.Q.; SY, A. and Liu, F.(1990): Antiinflammatory effects of verapamil, nifedipine and nicardipine. Zhongguo Yao Li Xue Bao. 11(3):281-285.

37- Kumar, A.; Azmatulla, A.; Babu, C.H.; Afroze, K.H. and Praveen, A. (2016): Anti-inflammatory effect of calcium channel blockers. In Rat Paw Edema Model. J. of Evidence Based Medicine and Healthcare. 14 (3): 493499.

38- Khaksari, M.; Mahani, S.E. and Mahmoodi, M. (2004): Calcium channel blockers reduce inflammatory edema in the rat: Involvement of the hypothalamus-pituitary-adrenal axis. Indian J of pharmaco. 36(6): 351-354. 